\title{
Intralayer magnetic ordering in Ge/Mn digital alloys
}

\author{
M. M. Otrokov, ${ }^{1}$ A. Ernst, ${ }^{2}$ S. Ostanin, ${ }^{2}$ G. Fischer, ${ }^{3}$ P. Buczek,${ }^{2}$ L. M. Sandratskii, ${ }^{2}$ W. Hergert,${ }^{3}$ I. Mertig,,${ }^{2,3}$ \\ V. M. Kuznetsov, ${ }^{1}$ and E. V. Chulkov ${ }^{4,5}$ \\ ${ }^{1}$ Physics Department, Tomsk State University, prospekt Lenina 36, 634050 Tomsk, Russia \\ ${ }^{2}$ Max-Planck-Institut für Mikrostrukturphysik, Weinberg 2, D-06120 Halle, Germany \\ ${ }^{3}$ Institut für Physik, Martin-Luther-Universität Halle-Wittenberg, D-06099 Halle, Germany \\ ${ }^{4}$ Departamento de Física de Materiales, Facultad de Ciencias Químicas, UPV/EHU and Centro de Física de Materiales \\ CFM-MPC, Centro Mixto CSIC-UPV/EHU, Apartado 1072, San Sebastián, 20080 Basque Country, Spain \\ ${ }^{5}$ Donostia International Physics Center (DIPC), Paseo de Manuel Lardizabal 4, San Sebastián, 20018 Basque Country, Spain
}

(Received 10 September 2010; revised manuscript received 17 December 2010; published 11 April 2011)

\begin{abstract}
We present a first-principles investigation of the electronic properties of $\mathrm{Ge} / \mathrm{Mn}$ digital alloys obtained by the insertion of $\mathrm{Mn}$ monolayers in the Ge host. The main attention is devoted to the study of the magnetic properties of the Mn layers for various types of ordering of the Mn atoms. Depending on the type of Mn position three different structures are considered: substitutional, interstitial, and combined substitutional-interstitial. In all three cases numerical structural relaxation of the atomic positions has been performed. We find that the intralayer exchange parameters depend strongly on the crystal structure. For the substitutional and interstitial types of structure the stable magnetic order was found to be ferromagnetic. For the mixed substitutional-interstitial structure the ferromagnetic configuration appears unstable and a complex ferrimagnetic structure forms. The spin-wave excitations are calculated within the Heisenberg model. The critical temperatures of the magnetic phase transitions are determined using Monte Carlo simulations with interatomic exchange parameters obtained for two different magnetic reference states: a ferromagnetic and a disordered local moment state.
\end{abstract}

DOI: 10.1103/PhysRevB.83.155203

PACS number(s): 75.70.Rf, 73.20.At, 68.37.Ef, 68.43.-h

\section{INTRODUCTION}

The spin-dependent electron-transport phenomena in diluted magnetic semiconductors (DMSs) make these systems an object of intensive theoretical and experimental studies. ${ }^{1,2}$ In the last decade, the research in this field was mostly focused on the III- and V-group-based DMSs, above all (Ga,Mn)As, where ferromagnetic (FM) ordering with relatively high Curie temperature up to $T_{\mathrm{C}}=185 \mathrm{~K}$ was observed. ${ }^{3-5}$ Unfortunately, rather low hole mobility of the order of $1-5 \mathrm{~cm}^{2} /(\mathrm{V} \mathrm{s})$ resulting from the Fermi level position within the impurity band is one of the serious obstacles in the way of (Ga,Mn)As-based spintronic devices. ${ }^{6,7}$ The group-IV DMSs received relatively low attention despite the possibility of their integration into mature silicon technology. This situation can be explained by technological difficulties in the preparation of homogeneously doped samples. There are several experimental reports on the high- $T_{\mathrm{C}}$ ferromagnetism in the group-IV DMSs. However, the physical mechanism of the formation of magnetic properties of these DMSs is far from being well understood. ${ }^{8-11}$ For instance, in $\mathrm{Ge}_{1-x} \mathrm{Mn}_{x}$ the coexistence of isolated Mn atoms, Mn-rich clusters, and $\mathrm{Mn}_{5} \mathrm{Ge}_{3}$ nanoparticles, dispersed across the Ge host, makes the magnetic behavior of the system extremely complex. ${ }^{12-14}$

Currently, the technique of $\delta$ doping allows DMSs of high quality and precisely controlled composition to be grown in the form of so-called digital magnetic alloys (DMAs). In a DMA, ultrathin $3 d$-metal layers can be periodically deposited inside the semiconductor host. The exchange coupling between the moments of the $3 d$ atoms in DMAs can be remarkably strong. ${ }^{15-17}$ Furthermore, the specific geometry of a DMA can prevent the in-plane diffusion of magnetic dopants, reducing the probability of their clusterization and secondary-phase formation. $\delta$ doping has been employed already to fabricate
(III,V)/Mn DMAs. ${ }^{18-23}$ The fabrication of $[\operatorname{Si}(20 \AA) / \operatorname{Mn}(x)]_{30}$ with the thickness $x=1-2 \AA$ has also been reported. ${ }^{32}$

All $a b$ initio studies performed up to now for the group-IV/3d DMAs show that $\mathrm{Mn}$ is the most promising dopant. ${ }^{15,24-30}$ For instance, when Mn substitutes Ge or Si within a single monolayer $(\mathrm{ML})\left(\mathrm{Mn}_{\mathrm{S}}\right)$, ferromagnetic order is expected and there is strong evidence for the formation of a half-metallic density of states. ${ }^{31}$ The morphology of the Mn ML seems to be a crucial factor which determines the magnetic properties of the system. Experiments on $\mathrm{Si} / \mathrm{Mn}$ reveal structural variations within $\delta$-doped layers. Molecular beam epitaxy produces $\delta$ layers with mostly substitutional $\mathrm{Mn}_{\mathrm{S}},{ }^{32}$ whereas the deposition of $\mathrm{Mn}$ onto the $\mathrm{Si}(001)-(2 \times 1)$ reconstructed surface leads to formation of a subsurface $\delta$ layer with manganese in interstitial positions $\left(\mathrm{Mn}_{\mathrm{I}}\right){ }^{33}$ which was found to be ferromagnetically ordered. $^{27}$

In this paper we present the results of an $a b$ initio study of $\mathrm{Ge} / \mathrm{Mn}$ DMAs. We focus on three structural compositions of the Mn ML. First, we consider the case of the $\mathrm{Mn}_{\mathrm{S}} \mathrm{ML}$, widely discussed in the literature (cf., e.g., Refs. 15, 24, and 25). Then we examine the interstitial $\mathrm{Mn}_{\mathrm{I}} \mathrm{ML}$ in Ge. Finally, we turn to the mixed substitutional-interstitial composition $\left(\mathrm{Mn}_{\mathrm{IS}} \mathrm{ML}\right)$. The latter is characterized by the double amount of manganese occupying both substitutional $\mathrm{Mn}_{\mathrm{S}}$ and interstitial $\mathrm{Mn}_{\mathrm{I}}$ sites in the $\delta$-doped layer. Since epitaxial growth is a nonequilibrium process, all three structural situations are in principle possible and should be considered. For each scenario, the electronic structure is calculated for numerically relaxed atomic positions. Next, the magnetic ordering is investigated based on the analysis of calculated parameters of the interatomic exchange coupling. Finally, we estimate the critical temperature for each system and calculate the spectrum of spin-wave excitations. 

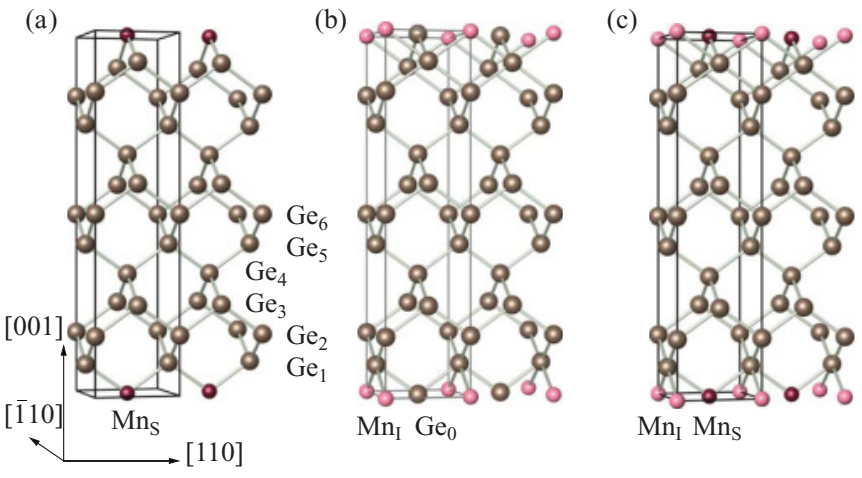

FIG. 1. Color $\mathrm{Ge} / \mathrm{Mn}$ unit cell with the substitutional $\mathrm{Mn}_{\mathrm{S}}$ (a), interstitial $\mathrm{Mn}_{\mathrm{I}}$ (b), and mixed $\mathrm{Mn}_{\mathrm{IS}}$ (c) ML. Large brown spheres represent $\mathrm{Ge}$, while small dark red and pink spheres represent $\mathrm{Mn}_{\mathrm{S}}$ and $\mathrm{Mn}_{\mathrm{I}}$, respectively. The Ge layers are labeled as shown in (a).

\section{DETAILS OF CALCULATION}

The Ge-based DMAs were modeled by tetragonal supercells, composed of the three different types of Mn ML and Ge spacer with a thickness of 11 atomic layers (see Fig. 1). The unit-cell basis vectors $\mathbf{a}, \mathbf{b}$, and $\mathbf{c}$ are directed along [110], [110], and [001], respectively, where $a=b=a_{\mathrm{Ge}} / \sqrt{2}$, $c=3 a_{\mathrm{Ge}}$, and $a_{\mathrm{Ge}}=5.77 \AA$ is the optimized lattice parameter of the diamond structure Ge.

We employed density functional theory within the generalized gradient approximation ${ }^{34}$ (GGA) to the exchangecorrelation potential and the projector augmented-wave method $^{35}$ implemented within the VASP code. ${ }^{36,37}$ To relax the atomic positions in the supercells we used a plane-wave basis set with a cutoff energy of $500 \mathrm{eV}$ and a $\Gamma$-centered mesh $6 \times 6 \times 1$ of special $\mathbf{k}$ points. ${ }^{38}$ The use of a more dense $\mathbf{k}$ mesh leads to marginal changes of the relaxed atomic positions, less than $0.25 \%$. All structural optimizations were performed until the forces on each atom were less than $10^{-2} \mathrm{eV} / \AA$.

For the relaxed geometry of $\mathrm{Ge} / \mathrm{Mn}$, further ab initio calculations were performed using multiple-scattering theory based on the Korringa-Kohn-Rostoker (KKR) method and the atomic sphere approximation to the crystal potentials. ${ }^{39-41}$ A complex energy contour integration with 24 Gaussian quadrature points was used, while for the Brillouin zone (BZ) integration a $24 \times 24 \times 6 \mathbf{k}$ mesh was utilized. The interatomic exchange parameters $J_{i j}$ of the Heisenberg Hamiltonian

$$
H=-\sum_{i, j} J_{i j} \mathbf{e}_{i} \cdot \mathbf{e}_{j}
$$

were evaluated using the magnetic force theorem (MFT) ${ }^{42}$ Here $i$ and $j$ number magnetic atoms, and $\mathbf{e}_{i}$ is a unit vector in the direction of the magnetic moment of the $i$ th atom. The exchange coupling parameters are calculated for both FM and disordered local moment ${ }^{43-45}$ (DLM) reference states. The DLM state models the system in the paramagnetic phase and is simulated within the framework of the coherent-potential approximation within the KKR Green's function method. ${ }^{46,47}$

In order to circumvent the well-known shortcoming of the GGA, which yields metallic behavior of bulk Ge, the $\mathrm{Ge}$ valence $p$ states were treated with an additional Coulomb potential $U \cdot{ }^{48,49}$ Thus, the calculated electronic spectrum of Ge was in good agreement with the spectrum obtained with the $G W$ method $^{50}$ which is known to reproduce experimental spectra well. ${ }^{51}$ With $U=1.53 \mathrm{eV}$ the calculated band gap is close to the experimental value of $0.65 \mathrm{eV}$.

To estimate the critical temperatures of the systems, Monte Carlo (MC) simulations were carried out (see Ref. 52 for details). Since we deal with two-dimensional layers of $\mathrm{Mn}$ atoms, it is important to relate our calculations to the conclusions of the Mermin-Wagner theorem. ${ }^{53,54}$ According to this theorem, the two-dimensional Heisenberg system without magnetic anisotropy does not feature a magnetically ordered phase at nonzero temperatures. The reason for the absence of long-range magnetic ordering in two dimensions is the Goldstone mode of the gapless long-wavelength magnetic excitations: the destructive role of this mode increases with decreasing dimensionality of the system. On the other hand, even a small gap in the excitation spectrum introduced by the magnetic anisotropy, present in any real two-dimensional system, can essentially reduce the impact of the low-energy excitations. In systems with a relatively large critical temperature, the magnetic ordering is governed by strong exchange interactions. The dependence of the critical temperature on the value of the magnetic anisotropy parameter has a weak logarithmic nature..$^{55}$ Therefore the variation of the magnetic anisotropy in a physically reasonable interval does not change the qualitative picture of the phase transformation. Our MC simulations were performed for the Hamiltonian

$$
H=-\sum_{i, j} J_{i j} \mathbf{e}_{i} \cdot \mathbf{e}_{j}-\sum_{i} \Delta\left(e_{i}^{z}\right)^{2},
$$

where $\Delta$ is the magnetic anisotropy parameter. The easy axis is taken along the $z$ axis. The simulations were performed for several values of $\Delta$.

During the simulation, Mn supercells with up to 7200 atoms were considered. The starting point was a high-temperature disordered state. In the course of the simulations the temperature was stepwise reduced until magnetic ordering was reached. For each temperature, the thermal equilibrium was assumed to be reached after $15 \times 10^{3} \mathrm{MC}$ steps. The thermal averages were determined over $25 \times 10^{3}$ MC steps.

The ground-state magnetic structure of a given system can in principle be determined by means of total-energy calcualtions for various magnetic states. However, in the case of systems with a large number of possible magnetic states this approach is associated with a vast computational effort. A more convenient method to determine the ground magnetic state is suggested by the evaluation of the effective parameters of the interatomic exchange interactions since they usually provide a reliable hint at the ground-state magnetic structure. In the context of MFT the exchange parameters can be calculated with respect to different reference states. In this paper we consider two reference states: ferromagnetic and paramagnetic. The paramagnetic state is described within the DLM picture. In an ideal Heisenberg system all calculations should lead to the same results. The difference of the exchange parameters calculated with respect to the mentioned reference states can be treated in terms of the temperature dependence of the effective interatomic exchange interactions. It is logical to 
expect that the parameters obtained for the DLM reference state are the better basis for the estimation of the critical temperature of the magnetic phase transition. ${ }^{57}$

Useful information on the stability of the FM state is also provided by the comparison of the energies of the FM and DLM states, $\Delta E=E_{\mathrm{DLM}}-E_{\mathrm{FM}}$, and by the analysis of the cumulative exchange parameter $J_{0}=\sum_{i \neq 0} J_{0 i}$. The value of $J_{0}$ describes the total exchange field at the position of a given atom resulting from the exchange interactions with all other magnetic atoms of the system.

In the case considered, similar values of the DLM-MFT and FM-MFT exchange parameters indicate their weak temperature dependence.

\section{RESULTS AND DISCUSSION}

In the DMAs the exchange coupling parameters strongly depend on the structural type of the Mn ML. They are also sensitive, although less dramatically, to the distances to the atoms of the spacer. Therefore, the optimization of the atomic positions in the DMAs is important. Since the bond lengths $\mathrm{Mn}-\mathrm{Ge}_{1}$ and $\mathrm{Mn}-\mathrm{Ge}_{2}$ are smaller than or comparable to the Mn-Mn distance in the ML, charge redistribution, caused by relaxations, could easily affect the exchange interactions in the Mn ML. To be specific, in the case of the $\mathrm{Mn}_{\mathrm{I}} \mathrm{ML}$ the bond length between $\mathrm{Mn}_{\mathrm{I}}$ and $\mathrm{Ge}_{1}, d_{\mathrm{Mn}_{\mathrm{I}}-\mathrm{Ge}_{1}}$, increases by $3.35 \%$ compared to that of Ge bulk, while $d_{\mathrm{Mn}_{\mathrm{I}} \mathrm{Ge}_{2}}$ is stretched by $5.16 \%$. Upon these structural changes, $J_{01}$ and $J_{02}$ were observed to increase by $40 \%$ and $125 \%$, respectively. In the Ge/Mn $/ \mathrm{MMA}_{\mathrm{S}} d_{\mathrm{Mn}_{\mathrm{S}}-\mathrm{Ge}_{1}}$

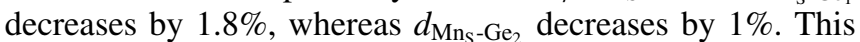
leads to $20 \%$ and $15 \%$ increase of $J_{01}$ and $J_{02}$, respectively. In the $\mathrm{Ge} / \mathrm{Mn}_{\mathrm{IS}}$ DMA relaxations are not so important, and $d_{\mathrm{Mn}_{(\mathrm{IS})}-\mathrm{Ge}_{1}}$ increases by just less than $1 \%$. Nevertheless, this changes $J_{02}^{\mathrm{I}-\mathrm{I}}$ by about $14 \%$. These results indicate a significant hybridization between $\mathrm{Mn}$ and Ge states. As a rule, relaxations in magnetic systems are determined by the electrostatic interaction, while the influence of the magnetic structure is much weaker. Below we discuss the results obtained with the structural relaxations performed for the FM ordering.

We begin with the consideration of the $\mathrm{Mn}_{\mathrm{S}} \mathrm{ML}$. For the FM reference state the nearest neighbors demonstrate robust ferromagnetic exchange with $J_{01}=25 \mathrm{meV}$ [Fig. 2(a)]. The coupling between more distant neighbors is much weaker, e.g., $J_{02}=-2 \mathrm{meV}$, while $J_{03}$ is close to $-6 \mathrm{meV}$. Other coupling parameters are negligibly small. The calculated cumulative exchange parameter $J_{0}=74.8 \mathrm{meV}$ and $\Delta E=$ $119 \mathrm{meV} /(\mathrm{Mn}$ atom $)$ are indications of the stability of the FM state for the substitutional type of Mn layer.

The magnetic moment on the $\mathrm{Mn}_{\mathrm{S}}$ atom is about $3.4 \mu_{\mathrm{B}}$. Taking into account the moments induced in the semiconductor host, the total moment per unit cell amounts to $3.04 \mu_{\mathrm{B}}$. These values and estimated exchange parameters are in a good qualitative agreement with the results obtained in Ref. 25.

Next, we consider the case of $\mathrm{Ge} / \mathrm{Mn}_{\mathrm{I}} \mathrm{DMA}$, where the impurity atoms are located at the same distances as in the $\mathrm{Mn}_{\mathrm{S}}$ ML case but have another atomic environment. The interaction between nearest neighbors, characterized by $J_{01} \simeq 16 \mathrm{meV}$ in FM-MFT, indicates a strong tendency toward FM ordering [Fig. 2(b)]. The coupling via the $\mathrm{Ge}_{0}$ atom (superexchange) with $J_{02}=3.7 \mathrm{meV}$ makes this trend even stronger. The
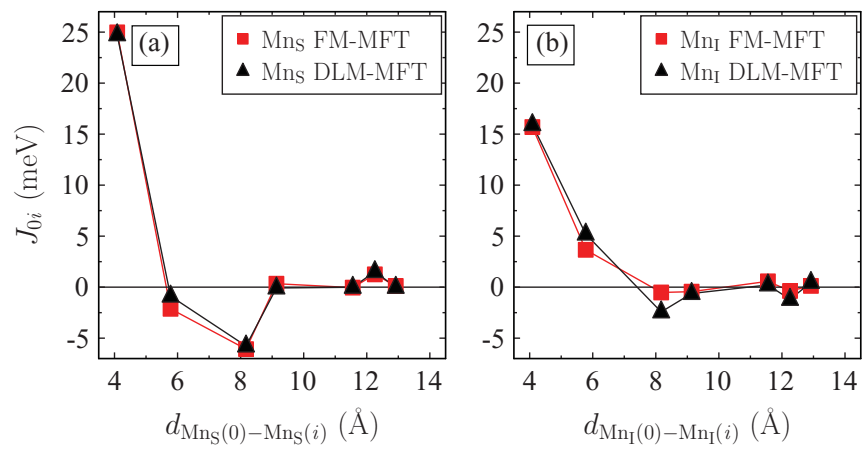

FIG. 2. Color Exchange coupling constants $J_{0 i}$ (in $\mathrm{meV}$ ) for $\mathrm{Ge} / \mathrm{Mn}_{\mathrm{S}}$ (a) and $\mathrm{Ge} / \mathrm{Mn}_{\mathrm{I}}$ (b), as a function of the $\mathrm{Mn}-\mathrm{Mn}$ distance. Red squares and black triangles correspond to the FM and DLM reference states, respectively.

exchange interactions with more distant neighbors are negligibly small. As a result, the cumulative exchange parameter $J_{0}=72.4 \mathrm{meV}$ and $\Delta E=81.4 \mathrm{meV} /(\mathrm{Mn}$ atom) indicate the FM ground state. The local and total magnetic moments in this case are equal to $3.46 \mu_{\mathrm{B}}$ and $3.41 \mu_{\mathrm{B}}$, respectively.

Note that for $\mathrm{Ge} / \mathrm{Mn}_{\mathrm{S}}$ and $\mathrm{Ge} / \mathrm{Mn}_{\mathrm{I}}$ systems the $\mathrm{FM}$ and DLM reference states yield very close values of the exchange coupling constants.

Regarding mixed $\mathrm{ML}, \mathrm{Mn}_{\mathrm{S}}$ and $\mathrm{Mn}_{\mathrm{I}}$ atoms have different atomic environments, cease to be equivalent and, therefore, have different values of atomic moments. Also the exchange interactions within $\mathrm{Mn}_{\mathrm{S}}$ and $\mathrm{Mn}_{\mathrm{I}}$ sublattices are different. In Fig. 3, we present the interaction parameters for the atoms of both sublattices with all neighboring atoms. The points common for both dependences correspond to the intersublattice interactions whereas different points correspond to the intrasublattice interactions.

The exchange coupling between the near neighbors is strongly negative which indicates instability of the ferromagnetic state in this case [see Fig. 3(a)]. This conclusion agrees with the total energy calculations, which show that (i) the energy of the DLM state is lower than the energy of the FM state and (ii) antiparallel alignment of the $\mathrm{Mn}_{\mathrm{S}}$ and $\mathrm{Mn}_{\mathrm{I}}$ atomic moments (ferrimagnetic state) has 374 and $158 \mathrm{meV}$ per $\mathrm{Mn}_{\mathrm{S}}-\mathrm{Mn}_{\mathrm{I}}$ pair lower energy than the parallel alignment (FM) and the DLM state, respectively. The exchange parameters calculated for the ferrimagnetic configuration are similar to those of the DLM state [except for the $J_{02}^{S-S}$ value; see Figs. 3(b) and 3(c)]. The exchange interaction between the neighboring substitutional and interstitial Mn atoms, $J_{01}^{\text {S-I }}$, is strongly negative, favoring the antiparallel alignment of the corresponding magnetic moments. The exchange parameters between the neighbors in the same sublattices, $J_{02}^{\mathrm{S}-\mathrm{S}}$ and $J_{02}^{\mathrm{I}-\mathrm{I}}$, are also negative, indicating that the system exhibits magnetic frustrations which can lead to more complex magnetic structure.

Figure 4 shows the calculated magnon spectra for $\mathrm{Mn}_{\mathrm{S}}$ and $\mathrm{Mn}_{\mathrm{I}}$ MLs in the FM and DLM reference states and for the ferrimagnetic $\mathrm{Mn}_{\mathrm{IS}}$ ML. As expected, the FM and DLM magnon spectra are almost identical for the $\mathrm{Mn}_{\mathrm{S}} \mathrm{ML}$, as well as for the $\mathrm{Mn}_{\mathrm{I}} \mathrm{ML}$. The positive magnon frequencies point clearly to a stable FM collinear magnetic structure. In the 

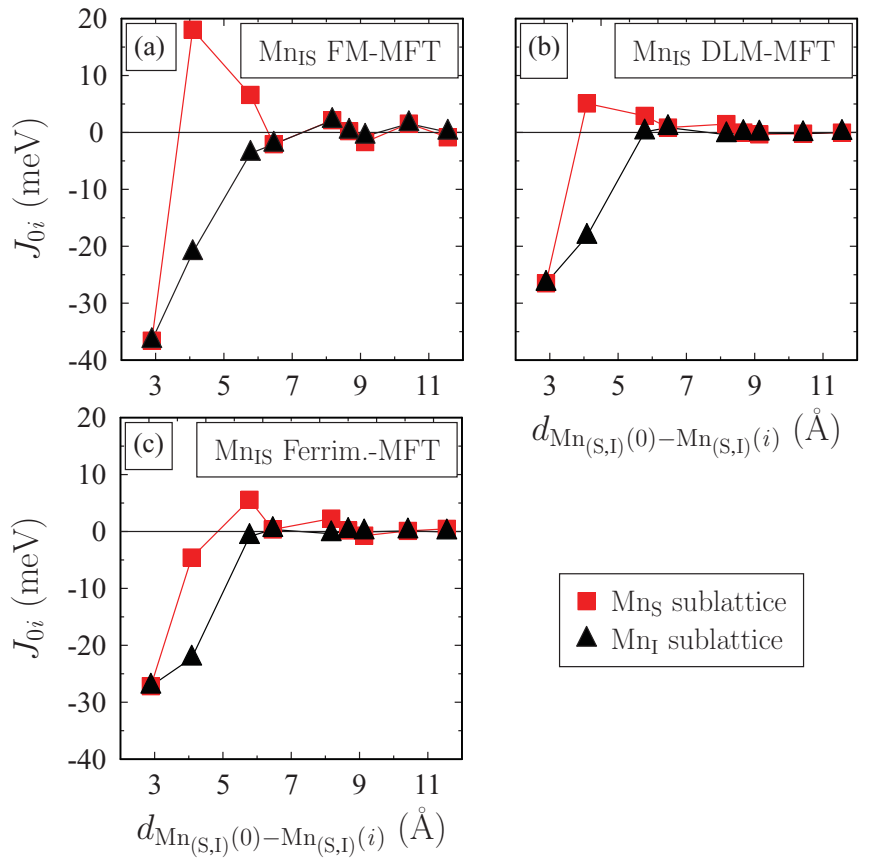

$d_{\mathrm{Mn}_{(\mathrm{S}, \mathrm{I})}(0)-\mathrm{Mn}_{(\mathrm{S}, \mathrm{I})}(i)}(\AA)$

Mns sublattice

$\boldsymbol{\Delta} \mathrm{Mn}_{\mathrm{I}}$ sublattice

FIG. 3. Color The same as Fig. 2, but for the $\mathrm{Mn}_{\mathrm{IS}}$ ML: (a) for the FM reference state in the $\mathrm{Mn}_{\mathrm{IS}} \mathrm{ML}$, (b) for the DLM reference state in the $\mathrm{Mn}_{\mathrm{IS}} \mathrm{ML}$, and (c) for the ferrimagnetic reference state (see explanations in text). Red squares correspond to the exchange coupling constants of $\mathrm{Mn}_{\mathrm{S}}$ atoms to its neighbors, and black triangles to those of $\mathrm{Mn}_{\mathrm{I}}$ atoms. The coinciding values of exchange constants correspond to intersublattice interactions, and the distinct ones to the intrasublattice coupling.
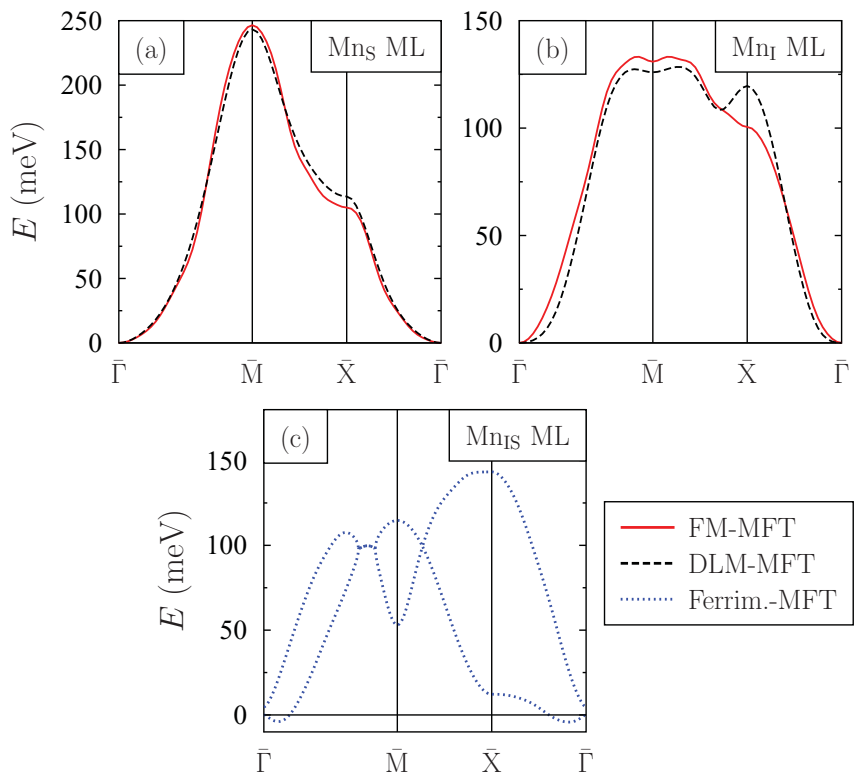

FIG. 4. (Color online) Calculated magnon spectra along $\bar{\Gamma}-\overline{\mathrm{M}}-$ $\overline{\mathrm{X}}-\bar{\Gamma}$ directions in the first two-dimensional $\mathrm{BZ}$ for $\mathrm{Ge} / \mathrm{Mn}_{\mathrm{S}}$ DMA (a), Ge/Mn $/ \mathrm{I}_{\mathrm{I}} \mathrm{DM}$ (b), and Ge/Mn $\mathrm{Mn}_{\mathrm{IS}} \mathrm{DMA}$ (c). The high-symmetry points have the following coordinates: $\bar{\Gamma},(0,0) ; \bar{M},\left(\pi / a_{\mathrm{Ge}}, \pi / a_{\mathrm{Ge}}\right)$; $\bar{X},\left(\pi / a_{\mathrm{Ge}}, 0\right)$. The red solid lines are obtained with the exchange parameters calculated for the FM reference state, and the black dashed lines with the exchange parameters for the DLM reference state; the blue dotted lines present the magnon spectra of the ferrimagnetic state of mixed $\mathrm{Mn}_{\mathrm{IS}}$ layer.
TABLE I. Critical temperatures $T_{\mathrm{C}}(\mathrm{K})$ estimated for different magnetic anisotropy energies $\Delta(\mathrm{meV})$ from DLM and FM reference states.

\begin{tabular}{ccccc}
\hline \hline & $\Delta$ & $\mathrm{Mn}_{\mathrm{S}} \mathrm{ML}$ & $\mathrm{Mn}_{\mathrm{I}} \mathrm{ML}$ & $\mathrm{Mn}_{\mathrm{IS}} \mathrm{ML}$ \\
\hline DLM & 0.5 & 208 & 178 & 280 \\
& 1.0 & 220 & 204 & 290 \\
FM & 0.5 & 152 & 256 & - \\
& 1.0 & 178 & 274 & - \\
\hline \hline
\end{tabular}

case of the ferrimagnetic $\mathrm{Mn}_{\mathrm{IS}} \mathrm{ML}$, there is a small region of long-wavelength magnons with slightly negative energy. This shows that in the system there is a weak instability with respect to formation of a noncollinear state. In this paper we do not investigate this issue further. It is possible that taking account of the magnetic anisotropy will stabilize the collinear ferrimagnetic state.

The critical temperatures obtained by means of $\mathrm{MC}$ simulations are summarized in Table I. It was found that coupling between Mn MLs is too weak to create magnetic interlayer ordering at temperatures significantly larger than zero kelvin. Therefore, the presented critical temperatures refer to the ordering within the Mn ML in the DMA. The values of the Curie temperature are rather high which is a consequence of the strong exchange interactions in the system. This shows the potential of digital doping for the fabrication of DMSs with high magnetic transition temperature. As expected, the influence of the parameter of magnetic anisotropy, $\Delta$, is relatively weak. Also the selection of the reference state in the calculation of the exchange parameters in the cases of the ferromagnetic $\mathrm{Mn}_{\mathrm{S}}$ and $\mathrm{Mn}_{\mathrm{I}}$ layers does not change the results qualitatively. From Table I it can be seen that an increase of $\Delta$ by a factor of 2 enlarges $T_{\mathrm{C}}$ by not more than $17 \%$.

\section{SUMMARY}

The magnetic ordering within Mn MLs of Ge/Mn was investigated within the density functional theory and Monte Carlo techniques. Using this approach, robust FM order was revealed for $\mathrm{Ge} / \mathrm{Mn}_{\mathrm{S}}$ and $\mathrm{Ge} / \mathrm{Mn}_{\mathrm{I}}$ DMAs. In the case of $\mathrm{Ge} / \mathrm{Mn}_{\mathrm{IS}}$, ferromagnetism is energetically not favorable, mainly because of the large negative exchange interactions between nearest neighbors of $\mathrm{Mn}_{\mathrm{S}}$ and $\mathrm{Mn}_{\mathrm{I}}$ atoms. In $\mathrm{Ge} / \mathrm{Mn}_{\mathrm{IS}}$, the Mn impurities occupy inequivalent crystallographic sites and feature different values of magnetic moments. We predict a complex ferrimagnetic ground-state spin configuration in this system.

\section{ACKNOWLEDGMENTS}

We acknowledge V. V. Tugushev for stimulating discussions. The work was partially supported by the Federal Targeted Program "Scientific and scientific-pedagogical personnel of innovative Russia in 2009-2013" and by the University of the Basque Country (Proyecto GV-UPV/EHU under Grant No. IT-366-07) and Spanish Ministerio de Ciencia y Tecnología (Grant No. FIS2010-19609-C02-01)]. Also we acknowledge the support by the Deutsche Forschungsgesellschaft DFG under Grant No. SFB 762 "Functionality 
of Oxidic Interfaces."The calculations were partially performed at SKIF Cyberia cluster in Tomsk, Russia and at Rechenzentrum Garching of the Max Planck Society (Germany).
${ }^{1}$ Semiconductor Spintronics and Quantum Computation, edited by D. D. Awschalom, D. Loss, and N. Samarth (Springer, New York, 2002).

${ }^{2}$ I. Žutić, J. Fabian, and S. Das Sarma, Rev. Mod. Phys. 76, 323 (2004).

${ }^{3}$ T. Jungwirth, J. Sinova, J. Mašek, J. Kučera, and A. H. MacDonald, Rev. Mod. Phys. 78, 809 (2006).

${ }^{4}$ K. Sato, L. Bergqvist, J. Kudrnovský, P. H. Dederichs, O. Eriksson, I. Turek, B. Sanyal, G. Bouzerar, H. Katayama-Yoshida, V. A. Dinh, T. Fukushima, H. Kizaki, and R. Zeller, Rev. Mod. Phys. 82, 1633 (2010).

${ }^{5}$ V. Novák, K. Olejník, J. Wunderlich, M. Cukr, K. Výborný, A. W. Rushforth, K. W. Edmonds, R. P. Campion, B. L. Gallagher, J. Sinova, and T. Jungwirth, Phys. Rev. Lett 101, 077201 (2008).

${ }^{6}$ K. S. Burch, D. B. Shrekenhamer, E. J. Singley, J. Stephens, B. L. Sheu, R. K. Kawakami, P. Schiffer, N. Samarth, D. D. Awschalom, and D. N. Basov, Phys. Rev. Lett. 97, 087208 (2006); K. Alberi, K. M. Yu, P. R. Stone, O. D. Dubon, W. Walukiewicz, T. Wojtowicz, X. Liu, and J. K. Furdyna, Phys. Rev. B 78, 075201 (2008).

${ }^{7}$ L. P. Rokhinson, Y. Lyanda Geller, Z. Ge, S. Shen, X. Liu, M. Dobrowolska, and J. K. Furdyna, Phys. Rev. B 76, 161201(R) (2007), and references therein.

${ }^{8}$ F. M. Zhang, X. C. Liu, J. Gao, X. S. Wu, Y. W. Du, H. Zhu, J. Q. Xiao, and P. Chen, Appl. Phys. Lett. 85, 786 (2004).

${ }^{9}$ M. Bolduc, C. Awo-Affouda, A. Stollenwerk, M. B. Huang, F. G. Ramos, G. Agnello, and V. P. LaBella, Phys. Rev. B 71, 033302 (2005).

${ }^{10}$ I. T. Yoon, C. J. Park, and T. W. Kang, J. Magn. Magn. Mater. 311, 693 (2007).

${ }^{11}$ A. F. Orlov, A. B. Granovsky, L. A. Balagurov, I. V. Kulemanov, Yu. N. Parkhomenko, N. S. Perov, E. A. Gan'shina, V. T. Bublik, K. D. Shcherbachev, A. V. Kartavykh, V. I. Vdovin, A. Sapelkin, V. V. Saraikin, Yu. A. Agafonov, V. I. Zinenko, A. Rogalev, and A. Smekhova, JETP 109, 602 (2009).

${ }^{12}$ C. Jaeger, C. Bihler, T. Vallaitis, S. T. B. Goennenwein, M. Opel, R. Gross, and M. S. Brandt, Phys. Rev. B 74, 045330 (2006).

${ }^{13}$ S. Ahlers, D. Bougeard, N. Sircar, G. Abstreiter, A. Trampert, M. Opel, and R. Gross, Phys. Rev. B 74, 214411 (2006).

${ }^{14}$ R. B. Morgunov, A. I. Dmitriev, and O. L. Kazakova, Phys. Rev. B 80, 085205 (2009).

${ }^{15}$ A. Continenza, F. Antoniella, and S. Picozzi, Phys. Rev. B 70, 035310 (2004).

${ }^{16}$ V. N. Men'shov, V. V. Tugushev, P. M. Echenique, S. Caprara, and E. V. Chulkov, Phys. Rev. B 78, 024438 (2008).

${ }^{17}$ V. N. Men'shov, V. V. Tugushev, S. Caprara, P. M. Echenique, and E. V. Chulkov, Phys. Rev. B 80, 035315 (2009).

${ }^{18}$ R. K. Kawakami, E. Johnston-Halperin, L. F. Chen, M. Hanson, N. Guébels, J. S. Speck, A. C. Gossard, and D. D. Awschalom, Appl. Phys. Lett. 77, 2379 (2000).

${ }^{19}$ T. C. Kreutz, G. Zanelatto, E. G. Gwinn, and A. C. Gossard, Appl. Phys. Lett. 81, 4766 (2002).

${ }^{20}$ B. D. McCombe, M. Na, X. Chen, M. Cheon, S. Wang, H. Luo, X. Liu, Y. Sasaki, T. Wojtowicz, J. K. Furdyna, S. J. Potashnik, and P. Schiffer, Physica E 16, 90 (2003).
${ }^{21}$ A. M. Nazmul, S. Kobayashi, S. Sugahara, and M. Tanaka, Physica E 21, 937 (2004).

${ }^{22}$ B. A. Aronzon, M. A. Pankov, V. V. Rylkov, E. Z. Meilikhov, A. S. Lagutin, E. M. Pashaev, M. A. Chuev, V. V. Kvardakov, I. A. Likhachev, O. V. Vihrova, A. V. Lashkul, E. Lähderanta, A. S. Vedeneev, and P. Kervalishvili, J. Appl. Phys. 107, 023905 (2010).

${ }^{23}$ S. V. Zaitsev, M. V. Dorokhin, A. S. Brichkin, O. V. Vikhrova, Yu. A. Danilov, B. N. Zvonkov, and V. D. Kulakovskii, JETP Lett. 90, 658 (2010).

${ }^{24}$ H.-Y. Wang and M. C. Qian, J. Appl. Phys. 99, 08 D705 (2006).

${ }^{25}$ S. Picozzi, M. Ležaić, and S. Blügel, Phys. Status Solidi A 203, 2738 (2006).

${ }^{26}$ M. C. Qian, C. Y. Fong, K. Liu, W. E. Pickett, J. E. Pask, and L. H. Yang, Phys. Rev. Lett. 96, 027211 (2006).

${ }^{27}$ H. Wu, P. Kratzer, and M. Scheffler, Phys. Rev. Lett. 98, 117202 (2007).

${ }^{28}$ Yu. A. Uspenskii and E. T. Kulatov, J. Magn. Magn. Mater. 321, 931 (2009).

${ }^{29}$ M. M. Otrokov, S. A. Ostanin, A. Ernst, V. M. Kuznetsov, and E. V. Chulkov, Phys. Solid State 52, 1680 (2010).

${ }^{30}$ C. Y. Fong, M. Shauhgnessy, R. Snow, and L. H. Yang, Phys. Status Solidi C 7, 747 (2010).

${ }^{31}$ S. Caprara, V. V. Tugushev, P. M. Echenique, and E. V. Chulkov, Europhys. Lett. 85, 27006 (2009).

${ }^{32}$ S. H. Chiu, H. S. Hsu, and J. C. A. Huang, J. Appl. Phys. 103, 07D110 (2008).

${ }^{33}$ M. R. Krause, A. J. Stollenwerk, J. Reed, V. P. La Bella M. Hortamani, P. Kratzer, and M. Scheffler, Phys. Rev. B 75, 205326 (2007).

${ }^{34}$ J. P. Perdew and Y. Wang, Phys. Rev. B 45, 13244 (1992).

${ }^{35}$ P. E. Blöchl, Phys. Rev. B 50, 17953 (1994).

${ }^{36}$ G. Kresse and J. Furthmüller, Phys. Rev. B 54, 11169 (1996).

${ }^{37}$ G. Kresse and D. Joubert, Phys. Rev. B 59, 1758 (1999).

${ }^{38}$ H. J. Monkhorst and J. D. Pack, Phys. Rev. B 13, 5188 (1976).

${ }^{39}$ J. Korringa, Physica (Amsterdam) 13, 392 (1947).

${ }^{40}$ W. Kohn and N. Rostoker, Phys. Rev. 94, 1111 (1954).

${ }^{41}$ M. Lüders, A. Ernst, W. M. Temmerman, Z. Szotek, and P. J. Durham, J. Phys.: Condens. Matter 13, 8587 (2001).

${ }^{42}$ A. I. Liechtenstein, M. I. Katsnelson, V. P. Antropov, and V. A. Gubanov, J. Magn. Magn. Mater. 67, 65 (1987).

${ }^{43}$ J. Hubbard, Phys. Rev. B 20, 4584 (1979).

${ }^{44}$ H. Hasegawa, J. Phys. Soc. Jpn. 46, 1504 (1979).

${ }^{45}$ B. L. Gyorffy, A. J. Pindor, J. Staunton, G. M. Stocks, and H. Winter, J. Phys. F: Met. Phys. 15, 1337 (1985).

${ }^{46}$ P. Soven, Phys. Rev. 156, 809 (1967).

${ }^{47}$ B. L. Gyorffy, Phys. Rev. B 5, 2382 (1972).

${ }^{48}$ V. I. Anisimov, J. Zaanen, and O. K. Andersen, Phys. Rev. B 44, 943 (1991).

${ }^{49}$ S. L. Dudarev, G. A. Botton, S. Y. Savrasov, C. J. Humphreys, and A. P. Sutton, Phys. Rev. B 57, 1505 (1998).

${ }^{50}$ D. Rideau, M. Feraille, L. Ciampolini, M. Minondo, C. Tavernier, H. Jaouen, and A. Ghetti, Phys. Rev. B 74, 195208 (2006). 
${ }^{51}$ Quasiparticle calculations in solids: W. G. Aulbur, L. Jönsson, and J. W. Wilkins, Solid State Phys. 54, 1 (2000).

${ }^{52}$ G. Fischer, M. Däne, A. Ernst, P. Bruno, M. Lüders, Z. Szotek, W. Temmerman, and W. Hergert, Phys. Rev. B 80, 014408 (2009).

${ }^{53}$ N. D. Mermin and H. Wagner, Phys. Rev. Lett. 17, 1133 (1966).

${ }^{54}$ M. Bander and D. L. Mills, Phys. Rev. B 38, 12015 (1988).
${ }^{55}$ P. Bruno, in Magnetic Surfaces, Thin Films and Multilayers, edited by S. S. P. Parkin et al., MRS Symposia Proceedings No. 231 (Materials Research Society, Pittsburgh, 1992), p. 299.

${ }^{56}$ M. Pajda, J. Kudrnovský, I. Turek, V. Drchal, and P. Bruno, Phys. Rev. Lett. 85, 5424 (2000).

${ }^{57}$ S. Shallcross, A. E. Kissavos, V. Meded, and A. V. Ruban, Phys. Rev. B 72, 104437 (2005). 